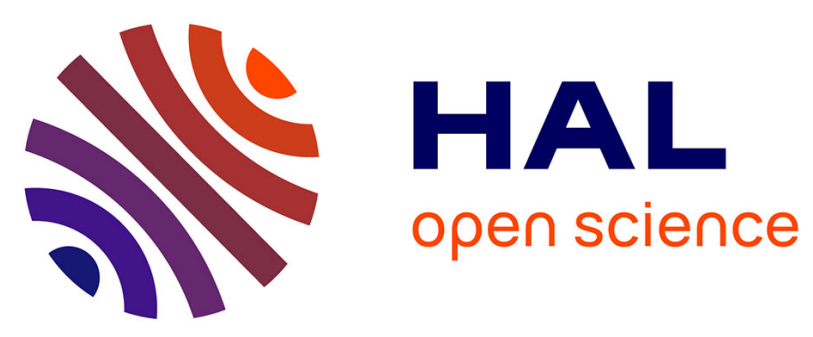

\title{
Automatic classification of skin lesions using color mathematical morphology-based texture descriptors
}

Victor Gonzalez-Castro, Johan Debayle, Yanal Wazaefi, Mehdi Rahim, Caroline Gaudy-Marqueste, Jean-Jacques Grob, Bernard Fertil

\section{- To cite this version:}

Victor Gonzalez-Castro, Johan Debayle, Yanal Wazaefi, Mehdi Rahim, Caroline Gaudy-Marqueste, et al.. Automatic classification of skin lesions using color mathematical morphology-based texture descriptors. Twelfth International Conference on Quality Control by Artificial Vision, Le2i - Laboratoire Electronique, Informatique et Image, Jun 2015, Le Creusot, France. pp.[9534-3]; doi:10.1117/12.2182592. emse-01163688

\section{HAL Id: emse-01163688 \\ https://hal-emse.ccsd.cnrs.fr/emse-01163688}

Submitted on 15 Jun 2015

HAL is a multi-disciplinary open access archive for the deposit and dissemination of scientific research documents, whether they are published or not. The documents may come from teaching and research institutions in France or abroad, or from public or private research centers.
L'archive ouverte pluridisciplinaire $\mathbf{H A L}$, est destinée au dépôt et à la diffusion de documents scientifiques de niveau recherche, publiés ou non, émanant des établissements d'enseignement et de recherche français ou étrangers, des laboratoires publics ou privés. 


\title{
Automatic classification of skin lesions using color mathematical morphology-based texture descriptors
}

\author{
Victor Gonzalez-Castro ${ }^{a}$, Johan Debayle ${ }^{a}$, Yanal Wazaefi ${ }^{b}$, Mehdi Rahim $^{b}$, Caroline \\ Gaudy-Marqueste ${ }^{c}$, Jean-Jacques $\mathrm{Grob}^{c}$ and Bernard Fertil ${ }^{b}$ \\ ${ }^{a}$ École Nationale Supérieure des Mines de Saint-Étienne, LGF UMR CNRS 5307, \\ Saint-Étienne, France; \\ ${ }^{b}$ Laboratoire des Sciences de l'Information et des Systems, UMR CNRS 7296, Marseille, \\ France; \\ ${ }^{c}$ Service de Dermatologie, Hôpital de la Timone, Marseille, France
}

\begin{abstract}
In this paper an automatic classification method of skin lesions from dermoscopic images is proposed. This method is based on color texture analysis based both on color mathematical morphology and Kohonen SelfOrganizing Maps (SOM), and it does not need any previous segmentation process. More concretely, mathematical morphology is used to compute a local descriptor for each pixel of the image, while the SOM is used to cluster them and, thus, create the texture descriptor of the global image. Two approaches are proposed, depending on whether the pixel descriptor is computed using classical (i.e. spatially invariant) or adaptive (i.e. spatially variant) mathematical morphology by means of the Color Adaptive Neighborhoods (CANs) framework. Both approaches obtained similar areas under the ROC curve (AUC): 0.854 and 0.859 outperforming the AUC built upon dermatologists' predictions (0.792).
\end{abstract}

Keywords: Melanoma, Color texture description, Mathematical morphology, Color adaptive neighborhoods, Self-organizing maps

\section{INTRODUCTION}

In 2012 there were more than 11150 new cases of skin cancer in France (3.1\% of all detected cancers), 15\% of which were mortal. Late diagnosis of skin cancer makes treatments much less efficient (i.e. a melanoma may become very aggressive in just a few months). Therefore, its early detection becomes essential to improve the chances of curing the skin cancer and, thus, the survival chances of the patient.

This is not an easy task for a non-experienced observer, as it is shown in figure 1. To carry out the detection of melanoma, dermatologists use several state-of-the-art methods, often called rules, such as the ABCD rule ${ }^{1}$ (Asymmetry, Border irregularity, Color irregularity and Differential structure, i.e. the size and number of structural features), the Menzies scoring namely the 7 -point checklist, ${ }^{2}$ which are based on the presence of certain texture patterns. Dermatologists detect malignancy features based on these rules and then combine this information to predict malignancy of the tumor.

An automation of such individual analysis of nevi would be very helpful not only for dermatologists, as they would make more accurate diagnosis and, thus, make better decisions about the need of surgery, but also for general practitioners, sending patients to the specialist more appropriately. Therefore, it would save economic

Further author information: (Send correspondence to Johan Debayle)

Victor Gonzalez-Castro: E-mail: victor.gonzalez@emse.fr

Johan Debayle: E-mail: debayle@emse.fr

Yanal Wazaefi: E-mail: yanal.wazaefi@gmail.com

Mehdi Rahim: E-mail: mehdi.rahim@lsis.org

Caroline Gaudy-Marqueste: E-mail: Caroline.Gaudy@ap-hm.fr

Jean-Jacques Grob: E-mail: jean-jacques.grob@ap-hm.fr

Bernard Fertil: E-mail: bernard.fertil@univ-amu.fr 


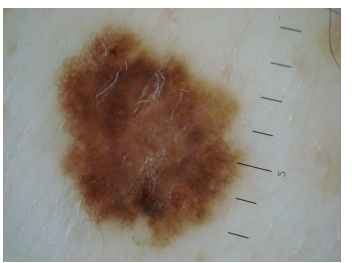

(a)

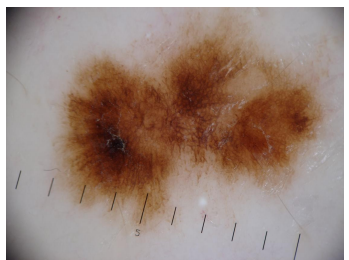

(b)

Figure 1. Example of an image of a benign lesion (a) and a melanoma (b).

and time resources to the Social Security system. Automatic melanoma detection - based on the analysis of dermoscopy images - has been receiving an increasing attention in the literature. ${ }^{3}$ Most works reproduce the classic rules used by dermatologists, extracting features linked to them. However, the extraction of such kind of features needs a previous segmentation of the lesion, which is often a very tricky step for computers, as it is demonstrated by the numerous papers devoted to automatic boundary detection of skin lesions ${ }^{4-6}$. Some examples of the reasons for such difficulty are: Low contrast between the lesion and the surrounding skin, irregular and fuzzy borders, fragmentation or variegated coloring inside the lesion.

In this paper an automatic classification method of skin lesions from dermoscopic images with no need of segmentation is proposed. It is based on a texture descriptor using color mathematical morphology ${ }^{7}$ and Kohonen self-organizing maps ${ }^{8}$ to characterize the image, as explained in section 2 . The performance of this descriptor has been assessed on a real dataset of skin lesions, as stated in section 3. Finally, conclusions and future perspectives are given in section 4 .

\section{THE PROPOSED TEXTURE DESCRIPTOR}

In this work, the texture of the color dermoscopic images of the skin lesions are described following a two-step procedure. First of all, all pixels are described using color features extracted by means of color mathematical morphology, which were already used in the experiments presented in. ${ }^{7}$ Afterwards, these pixel descriptors are used as input for a Kohonen self-organizing map to cluster them and, finally, the histogram of the cluster occurrences is used as global image descriptor. This procedure avoids the segmentation of the lesion, which is difficult to be carried out properly.

\subsection{Local pixel descriptor}

\subsubsection{Color mathematical morphology}

Morphological operators ${ }^{9}$ need the sets of the intensities to be processed to hold a total order relationship. However, in the case of color images this is not straightforward due to the vectorial nature of their points.

In the literature several order relationships have been proposed (i.e., marginal, lexicographical, partial or reduced ordering). In this work the so-called $\Omega$-ordering - denoted as $\prec_{\Omega}-$ proposed by Angulo ${ }^{10}$, has been used:

$$
c_{1} \prec_{\Omega} c_{2}=\left\{\begin{array}{l}
d_{R G B}\left(c_{1}, c_{0}\right)>d_{R G B}\left(c_{2}, c_{0}\right) \text { or } \\
d_{R G B}\left(c_{1}, c_{0}\right)=d_{R G B}\left(c_{2}, c_{0}\right) \text { and } \\
\left\{\begin{array}{l}
c_{1}^{R}<c_{2}^{R} \text { or } \\
c_{1}^{R}=c_{2}^{R} \text { and } c_{1}^{G}<c_{2}^{G} \text { or } \\
c_{1}^{R}=c_{2}^{R} \text { and } c_{1}^{G}=c_{2}^{G} \text { and } c_{1}^{B}<c_{2}^{B}
\end{array}\right.
\end{array}\right.
$$

where $d_{R G B}\left(c_{1}, c_{2}\right)$ represents the distance between two points $c_{1}=\left(c_{1}^{R}, c_{1}^{G}, c_{1}^{B}\right)$ and $c_{2}=\left(c_{2}^{R}, c_{2}^{G}, c_{2}^{B}\right)$ in the color space RGB (see equation 2) and $c_{0}$ stands for the reference color, which in this work has been set to $c_{0}=(0,0,0)$. It is easy to prove that $\prec_{\Omega}$ is a total order relationship. This order can be used with any color space. More details about color ordering can be found in $^{7}$. 


$$
d_{R G B}\left(c_{1}, c_{2}\right)=\sqrt{\left(c_{1}^{R}-c_{2}^{R}\right)^{2}+\left(c_{1}^{G}-c_{2}^{G}\right)^{2}+\left(c_{1}^{B}-c_{2}^{B}\right)^{2}}
$$

Let $x$ be a point of the spatial support $D \subseteq \mathbb{R}^{2}$, the resulting classical color erosion and dilation of an image f : $D \rightarrow \mathbb{R}^{3}$ at point $x$ by means of a disk $B_{r}$ of radius $r$ as structuring element (SE) is given respectively by:

$$
\begin{aligned}
& E_{r}(f)(x)=\inf _{\Omega}\left\{f(w): w \in B_{r}(x)\right\} \\
& D_{r}(f)(x)=\sup _{\Omega}\left\{f(w): w \in \check{B}_{r}(x)\right\}
\end{aligned}
$$

where $\sup _{\Omega}$ and $\inf _{\Omega}$ stand for the supremum and infimum according to the total order relationship $\prec \Omega$. It is possible to define more advanced operators by combining dilations and erosions such as openings, closings alternate filters, toggle contrast, top hat, etc.

\subsubsection{Color adaptive mathematical morphology}

The idea behind adaptive mathematical morphology is to replace the classical spatially invariant (i.e. with fixed shape and size) SE by spatially variant (i.e. adaptive) SEs. In this way, the Color Adaptive Neighborhoods $(\mathrm{CANs})$ - an extension to color of the General Adaptive Neighborhoods (GANs) ${ }^{11,12}-$, defined in, ${ }^{7}$ can be used as adaptive structuring element for color morphological operators. The Color Adaptive Neighborhood (CAN) of a point $x$, denoted $V_{m}^{f}(x)$, is defined as a spatial region included in $D$ built upon the color image $f$ in relation with a homogeneity tolerance value $m \in \mathbb{R}^{+}$. More precisely, $V_{m}^{f}(x)$ must fulfill two conditions:

- its points must have a color value close to the one of $x$, and

- the set has to be path-connected, considering the usual Euclidean topology on $D \subseteq \mathbb{R}^{2}$.

Therefore, CANs are formally defined as:

$$
V_{m}^{f}(x)=C_{\left\{y \in D ; d_{R G B}(f(y), f(x)) \leq m\right\}}(x)
$$

where $C_{X}(x)$ denotes the path-connected component of $X$ which contains $x \in D$, and $d_{R G B}(f(y), f(x))$ stands for the distance between the color points $f(y)$ and $f(x)$ in the color space RGB.

However, if they were used directly as Adaptive Structuring Elements (ASEs), the symmetry property, i.e. $x \in V_{m}^{f}(y) \Longleftrightarrow y \in V_{m}^{f}(x)$ - which is relevant for visual, topological, morphological and practical reasons ${ }^{11}$ - might not be satisfied. Therefore, the so-called Strong Color Adaptive Neighborhoods (S-CANs) are used as ASEs:

$$
N_{m}^{f}(x)=\bigcup_{z \in D}\left\{V_{m}^{f}(z) \mid x \in V_{m}^{f}(z)\right\}
$$

Thus, the elementary adaptive morphological operators erosion and dilation are defined respectively as:

$$
\begin{gathered}
E_{m}(f)(x)=\inf _{\Omega}\left\{f(w): w \in N_{m}^{f}(x)\right\} \\
\left.D_{m}(f)(x)=\sup _{\Omega}\left\{f(w): w \in N_{m}^{f}(x)\right)\right\}
\end{gathered}
$$




\subsubsection{Pixel-level description}

The descriptor of each pixel is formed by a concatenation of (i) its color components in the original image and (ii) the color components of that point in multiscale dilations and erosions (i.e., using structuring elements of different sizes $p_{i}$ ). For classical (resp. adaptive) mathematical morphology, $p_{i}$ denotes the radius (resp. homogeneity tolerance) of the SE (resp. adaptive structuring element).

Therefore, the general expression (i.e. for either classical or adaptive mathematical morphology) of the descriptor of each pixel $x$ in $D$ is:

$$
X_{n}(x)=\left[E_{p_{n}}(f)(x), \ldots, E_{p_{1}}(f)(x), f(x), D_{p_{1}}(f)(x), \ldots, D_{p_{n}}(f)(x)\right]
$$

where $n$ stands for the number of erosions and dilations which are carried out, $f(x)$ represents the values of the color components of the image $f$ at $x$ and $E_{p_{i}}(f)(x)$ (resp. $D_{p_{i}}(f)(x)$ ) represents the values of the color components at $x$ of the erosions (resp. dilations) of $f$ with a structuring element with size or tolerance $p_{i}$. Figure 2 shows an illustration of such concatenation.

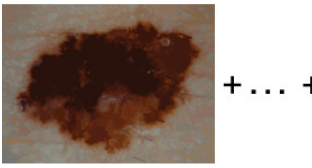

Erosion $n$

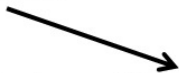$$
X_{n}(x)=\left[E_{p_{n}}(f)(x), \ldots, E_{p_{1}}\right.
$$

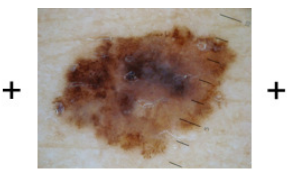

Original image

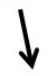

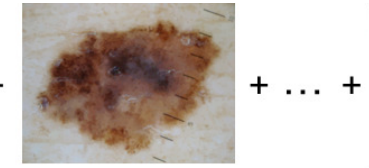

Dilation 1

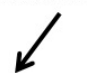

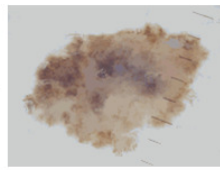

Dilation $n$

Figure 2. Illustration of the concatenation of color components to form the pixel descriptor using color adaptive mathematical morphology.

\subsection{Global image descriptor}

Once the pixels of an image are described, a global descriptor of the image is built. This is done by means of a Kohonen Self-Organising Map. ${ }^{8}$ First of all, 10 representative images of each class were selected by experts, and the lesions were roughly segmented manually. Thereafter, the map was trained using the pixels of the lesions and $5 \%$ of the other pixels (randomly selected). Note that this segmentation is only used for these 20 training images.

Afterwards, the pixels of the image are projected onto the neurons of the map in order to cluster them. Finally, the final image descriptor is the histogram of the hits of the neurons, i.e. the number of pixels that have been projected on each neuron of the map.

A diagram showing the description process is shown in figure 3 .

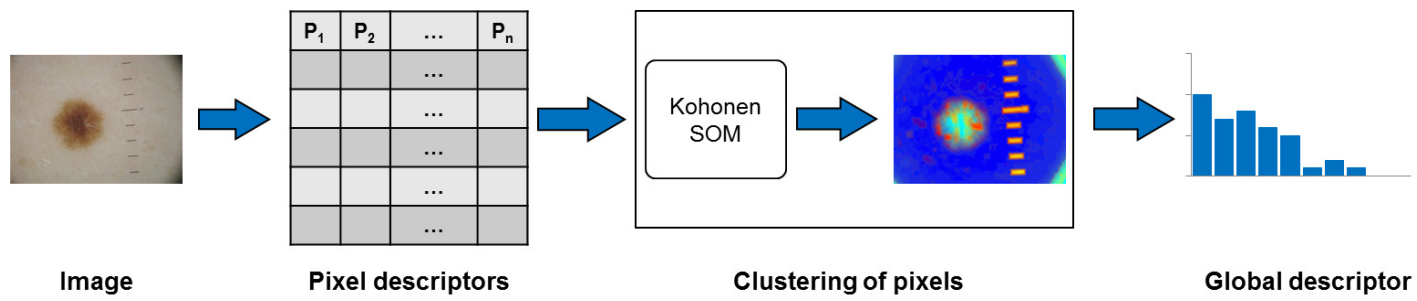

Figure 3. Diagram of the image description process. 


\section{EXPERIMENTS AND RESULTS}

\subsection{Experiments}

\subsubsection{Image database}

The image dataset that have been used in this experiment was composed of 1097 dermoscopic images of pigmented skin lesions, 88 of them being histopathologically confirmed melanomas. Thus, two classes were considered in this experiment: on the one hand confirmed melanomas and on the other hand the remaining benign lesions.

All images were acquired by several dermatologists equipped by a digital camera (SONY W120) combined with a Heine Delta 20 dermoscope.

\subsubsection{Image descriptor}

The local pixel descriptor described in section 2.1.3 has been computed using (i) classical (i.e. non adaptive) erosions and dilations and (ii) the adaptive CAN-based erosions and dilations, for the sake of comparison. In the case of the classical erosions and dilations, a square-shaped structuring element has been used, with width sizes varying between 5 and 50 in steps of 5 . Therefore, 10 different erosions and dilations were carried out. In the case of the adaptive CAN-based erosions and dilations, the values for the tolerance $m$ varied from 5 to 50 in steps of 5 . Therefore, in both cases, each pixel was described by means of 63 features (i.e. the RGB components of the pixel in the original image and its components after the erosions and dilations).

Afterwards, a Kohonen map of size $20 \times 20$ was used as explained in section 2.2. Therefore, each image has been described by means of a feature vector of length 400 .

\subsubsection{Classification}

Images were subsequently classified by means of a feed-forward Artificial Neural Network (ANN) working on their feature vectors. The data were normalized before classification, so that they had mean zero and standard deviation one. In this experiment a network with one hidden layer and a logistic sigmoid activation function for the hidden and output layers have been employed. The learning of the network was carried out with the momentum and adaptive learning rate algorithm. Different combinations of training cycles and neurons in the hidden layer have been used, in order to assess the impact of this configuration on the results. Results presented here correspond to the best configuration for each approach (classical and adaptive).

The classification was carried out using 10-fold cross validation, and the process was repeated 10 times, in order to avoid possible random effects (e.g. due to the random initialization of the network) and overfitting. The presented results are an average of these 10 runs.

\subsection{Results}

The receiver operating characteristics (ROC) curves $^{13}$ of the classifications corresponding to the best configuration of cycles-neurons (i.e. the ones that achieved the best results) are shown in figure 4. These configurations were 300 cycles and 7 neurons, and 400 cycles and 9 neurons for the classical and adaptive approaches, respectively. The ROC curve is more suitable to illustrate the performance of a classifier than the accuracy of the classification, and it is widely used in visualizing and analyzing the behavior of diagnostic systems. It depicts relative tradeoffs between benefits (true positives, or sensitivity) and costs (false positives, or 1-specificity). More details about ROC curves can be found in. ${ }^{13}$

Overall, both classifiers are comparable. The classifier generated by means of the descriptor based on classic mathematical morphology slightly outperforms the CAN-based one, obtaining areas under the curve (AUC) of 0.859 and 0.854 , respectively. A mean ROC curve, estimated in ${ }^{14}$ from the predictions carried out by nine dermatologists with this same image dataset, is also depicted in figure 4. This curve has an AUC of 0.792. Therefore, the descriptors proposed in this paper generated, without any segmentation step, classifiers which outperformed the actual predictions of the dermatologists. In particular, the classifiers were found particularly efficient in the distal part of the ROC curves, where the detection of the remaining melanoma is difficult. 


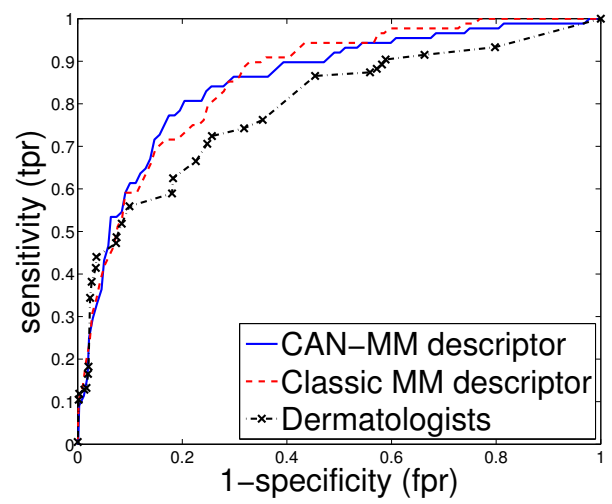

Figure 4. ROC Curves of the classifiers generated by means of the Classic Mathematical Morphology and the CAN-based Mathematical Morphology, together with the mean ROC curve observed for the dermatologists.

\section{CONCLUSIONS AND PERSPECTIVES}

In this work, a color texture descriptor has been introduced and applied to the description and classification of images of nevi as benign lesions or melanoma. The texture descriptor is built from local color data of each pixel, obtained from mathematical morphology operations. It is afterwards used to globally describe the image, by means of Kohonen Self-Organizing Maps.

Two approaches of this descriptor have been assessed in this work: (i) the local data has been obtained using classic (i.e. non adaptive) color mathematical morphology operations and (ii) getting this local data by means of adaptive color mathematical morphology based on the CAN framework. Both the classic and the adaptive approaches outperformed the dermatologists' predictions on the same image dataset, which obtained mean ROC curve with an AUC of $0.792^{14}$ achieving AUCs of 0.859 and 0.854 , respectively.

For future work, other features derived from the CANs ${ }^{15}$ - e.g. geometrical or morphometrical features to build the local pixel descriptor will be tackled. In addition, other different texture approaches combining CAN-based features with Local Binary Patterns can be assessed.

\section{REFERENCES}

[1] Nachbar, F., Stolz, W., Merkle, T., Cognetta, A. B., Vogt, T., Landthaler, M., Bilek, P., Braun-Falco, O., and Plewig, G., "The ABCD rule of dermatoscopy: High prospective value in the diagnosis of doubtful melanocytic skin lesions," Journal of the American Academy of Dermatology 30, 551-559 (apr 1994).

[2] Johr, R. H., "Dermoscopy: alternative melanocytic algorithms - the ABCD rule of dermatoscopy, menzies scoring method, and 7-point checklist," Clinics in Dermatology 20, 240-247 (may 2002).

[3] Korotkov, K. and Garcia, R., "Computerized analysis of pigmented skin lesions: A review," Artificial Intelligence in Medicine 56(2), 69 - 90 (2012).

[4] Glaister, J., Wong, A., and Clausi, D., "Segmentation of skin lesions from digital images using joint statistical texture distinctiveness," Biomedical Engineering, IEEE Transactions on 61, 1220-1230 (April 2014).

[5] Celebi, M., Iyatomi, H., Schaefer, G., and Stoecker, W. V., "Lesion border detection in dermoscopy images," Computerized Medical Imaging and Graphics 33(2), 148 - 153 (2009).

[6] Erkol, B., Moss, R. H., Joe Stanley, R., Stoecker, W. V., and Hvatum, E., "Automatic lesion boundary detection in dermoscopy images using gradient vector flow snakes," Skin Research and Technology 11(1), 17-26 (2005).

[7] González-Castro, V., Debayle, J., and Pinoli, J.-C., "Color Adaptive Neighborhood Mathematical Morphology and its application to pixel-level classification," Pattern Recognition Letters 47, 50-62 (oct 2014).

[8] Kohonen, T., [Self-Organizing Maps], vol. 30 of Springer Series in Information Sciences, Springer Berlin Heidelberg (1997).

[9] Serra, J., [Image Analysis and Math. Morphology], Academic Press (1982). 
[10] Angulo, J., "Morphological colour operators in totally ordered lattices based on distances: Application to image filtering, enhancement and analysis," Computer Vision and Image Understanding 107(1 - 2), 56 - 73 (2007).

[11] Debayle, J. and Pinoli, J.-C., "General Adaptive Neighborhood Image Processing: Part I: Introduction and Theoretical Aspects," Journal of Mathematical Imaging and Vision 25(2), 245-266 (2006).

[12] Debayle, J. and Pinoli, J.-C., "General Adaptive Neighborhood Image Processing: Part II: Practical Application Examples," Journal of Mathematical Imaging and Vision 25(2), 267-284 (2006).

[13] Fawcett, T., "An introduction to ROC analysis," Pattern Recognition Letters 27, 861-874 (jun 2006).

[14] Wazaefi, Y., Paris, S., and Fertil, B., "Contribution of a classifier of skin lesions to the dermatologist's decision," in [Image Processing Theory, Tools and Applications (IPTA), 2012 3rd International Conference on], 207-211 (Oct 2012).

[15] Rivollier, S., Debayle, J., and Pinoli, J.-C., "Adaptive shape diagrams for multiscale morphometrical image analysis," Journal of Mathematical Imaging and Vision 49(1), 51-68 (2014). 